\title{
Maternal and fetal outcome of pregnancy complicated by convulsions
}

\author{
Michelle Fonseca, Aditi Joshi* \\ Department of Obstetrics and Gynecology, Lokmanya Tilak Municipal General Hospital and Medical College,
} Mumbai, Maharashtra, India

Received: 24 June 2018

Accepted: 26 July 2018

\section{*Correspondence:}

Dr. Aditi Joshi,

E-mail: avj111@gmail.com

Copyright: $\odot$ the author(s), publisher and licensee Medip Academy. This is an open-access article distributed under the terms of the Creative Commons Attribution Non-Commercial License, which permits unrestricted non-commercial use, distribution, and reproduction in any medium, provided the original work is properly cited.

\begin{abstract}
Background: Convulsions in pregnancy pose serious morbidity and mortality to both mother and the fetus. Eclampsia being the most common cause. However, many medical disorders like epilepsy, cerebral malaria, meningitis, metabolic disorders etc can result in convulsions in pregnancy. This study was conducted to determine the prevalence of various aetiologies leading to convulsions and to assess how the various demographic-clinical characteristics affect the maternal as well as fetal outcome in patients with eclampsia as compared to those with convulsions other than eclampsia.

Methods: This was an observational study conducted over a period of one and a half year at a tertiary institute in India. A total of 56 patients were studied in this duration. The primary objective was to determine the prevalence of the aetiologies causing convulsion as well as the maternal and fetal outcome of the pregnancy complicated by convulsions. The data regarding age, parity, mode of delivery, gestational age at birth, risk factors was obtained by a predesigned proforma.

Results: A total of 56 patients were included in the study after screening 18,840 patients in the hospital. Hence the estimated prevalence of convulsions in pregnancy was 1:336 in this hospital. The most common aetiology noted was Eclampsia (69.6\%) followed by epilepsy (16.1\%). Others included Cerebral Malaria (3.6\%), Hypocalcemia (3.6\%), Neurocysticerosis (2\%), Hypokalemia (1.8\%) and Hypoglycemia (1.8\%). Maternal case fatality rate was 3.6\%. Total perinatal mortality was $14.2 \%$ of which $6(10.7 \%)$ were still birth.

Conclusions: Eclampsia is a major cause of convulsions in pregnancy and considered to be the diagnosis unless proved otherwise. However other causes of convulsions should not be ignored. A thorough evaluation of the patient which lays a major emphasis on a detailed history taking and examination, form the crux of diagnosing the cause of convulsions and can expediate a timely and accurate treatment.
\end{abstract}

Keywords: Anti-epileptic drugs, Convulsions, Epilepsy, Eclampsia, Non-eclamptic seizures, Obstetric outcomes

\section{INTRODUCTION}

The Four Horsemen of Death in the late 20th century were Convulsions, Puerperal Pyrexia, Haemorrhage and illegal abortion. These still remain the leading causes of maternal mortality in developing countries like India. Eclampsia is the leading cause of convulsions accounting for upto $12 \%$ of maternal deaths. ${ }^{1}$
Other causes of convulsions in pregnancy include epilepsy, hypertensive vascular diseases (Intracranial haemorrhage, thrombosis), cortical sinus thrombosis, metabolic causes: e.g. Hypoglycemia, hyponatremia, hypernatremia, hypocalcemia, uremia, Intracranial Space Occupying lesions: e.g. Brain tumours, Tuberculoma, infections: e.g. malaria, meningitis, neurocysticercosis, Injuries and drug reactions: e.g. Lignocaine toxicity. 
Epilepsy is the most common non-eclamptic cause of convulsion in pregnancy. Pregnancy- induced change in the antiepileptic drug metabolism and an increased hepatic and renal clearance are major factors affecting seizure control during pregnancy, although compliance is also a significant contributory factor. Seizure frequency remains unchanged during pregnancy in around $50 \%$, it may increase in up to $30 \%$ and decrease in up to $20 \%$. About $1-2 \%$ women with epilepsy can have a convulsion in labour or within the first 24 hours after delivery. ${ }^{2}$

Women with epilepsy have an increased risk of certain obstetrical complications. There is an approximately twofold increased risk of vaginal bleeding, anaemia, hyperemesis gravidarum, abruptio placentae, eclampsia, premature rupture of membranes, induced labour, and cesarean section. ${ }^{3}$

The overall incidence of fetal malformations noted in newborns is around 5-6\%. This risk of malformations increases with increase in the number of medications, rising upto almost $10-20 \%$ with three anti-epileptics. Babies born to women with epilepsy on treatment are at an increased risk for intrauterine growth retardation, major congenital malformations, minor anomalies, microcephaly, cognitive dysfunction, long term neurological sequelae or at times infant mortality. ${ }^{3,4}$

A comprehensive pre-conceptional counselling is the crux of management in these patients. Folate supplementation pre-conceptionally is ideal to prevent neural tube defects. Although there is an approximately two-fold increase in chance of interventions during labour and delivery, most women with epilepsy have a safe vaginal delivery. Sleep deprivation, prolonged labour, erratic absorption of anti-epileptic drugs further aggravated by emesis are some of the factors precipitating seizures during labour. Perinatal lethargy, irritability, and feeding difficulties have been attributed to intrauterine exposure to anti-epileptic drugs (AED), especially with barbiturates and benzodiazepines. The puerperium with its inevitable sleep disruption is often a time of seizure worsening, including seizure recurrence for some women with previously controlled seizures. Hence extra vigilance is required at this time.

There are other causes of convulsions also. Considering the common occurrence of not only eclamptic convulsions, but also those of other origin, this study was conducted to look at the outcomes - medical and obstetric. It was hypothesized that a good number of convulsions apart from eclampsia would be encountered and that the outcomes in non-eclamptic patients would be different from those with eclampsia.

\section{METHODS}

This was a prospective observational study conducted from January 2015- June 2016 at the Lokmanya Tilak
Municipal Medical College and General Hospital, Sion, Mumbai.

A total of 56 patients presenting with convulsions in pregnancy were studied. Data pertaining to maternal age, parity, mode of delivery, maternal and fetal outcome was obtained via a predesigned case proforma.

On admission a thorough history was taken and a detailed clinical examination was carried out which included examination of pulse, blood pressure, pallor, icterus, fetal heart sounds, per abdomen examination, abdominal palpation, per speculum and per vaginum examination and central nervous system examination.

All the patients were subjected to biochemical investigations including complete blood count, HIV, HBsAg, VDRL test, Liver function tests (Serum bilirubin, SGOT, SGPT, alkaline phosphatase) Renal function tests (serum creatinine, Blood urea, serum uric acid), serum electrolytes and Obstetric ultrasound.

The various diagnostic and therapeutic modalities used for management included malarial antigen, dengue antibody and leptospirosis antibody in suspected cases of acute febrile illness. Prothrombin time, activated partial thromboplastin time, serum fibrinogen and D-dimer levels in cases where coagulation abnormality was suspected. CT Scan of Brain was done post-delivery in selective cases. EEG was done in few selected cases.

The course of pregnancy was studied, and the investigation profile was monitored.

The treatment of the study population was at the discretion of the attending physician. Anti- hypertensive, anticonvulsants and necessary obstetric management was used in cases of eclampsia. Anti-epileptic medications were given to epileptic patients. Blood and blood products (Platelets, fresh frozen plasma and cryoprecipitate) were transfused as per deficiency and associated clinical evaluation.

Critically ill patients were managed in medical intensive care unit and specialized measures like mechanical ventilator were given to indicated patients. In case of a maternal death, autopsy was performed whenever required and findings were studied. In maternal outcome, the condition of mother at discharge, mode of delivery and course of stay in hospital was noted. For fetal outcome, mode of delivery, NICU requirement and perinatal mortality was noted.

\section{Inclusion criteria}

- All patients presenting for the first time with convulsion in pregnancy or had been previously diagnosed with epilepsy or with history of convulsion in present or past associated with known 
h/o of brain tumour/injury/ metabolic disorder/ neurological disorder/ meningitis/ cerebral malaria.

\section{Exclusion criteria}

Those unwilling to consent for procedure.

\section{Statistical analysis}

Final outcome of all cases and complications if any was statistically analyzed. Data were statistically described in terms of mean $( \pm \mathrm{SD})$, frequencies (number of cases) and percentages when appropriate. Data were tested first for normal distribution by Klomogorov- Smirnov test. Comparison of quantitative variables between the study groups was done using Student $t$ test for independent samples if normally distributed. Mann-Whitney U test was used for non-normally distributed quantitative data. For comparing categorical data, Chi square test was performed. Exact test was used instead when the expected frequency is less than 5. All statistical calculations were done using computer programs Microsoft Excel 2007 (Microsoft Corporation, NY, USA) and SPSS (Statistical Package for the Social Science; SPSS Inc., Chicago, IL, USA) version 21.

\section{RESULTS}

This study included 56 patients who satisfied the inclusion criteria after screening 18,840 patients in the hospital during the given period. Hence the estimated prevalence of convulsions in pregnancy was approximately 1:336. The prevalence of convulsions in India varies according to the aetiology of the convulsion. In this study the prevalence was 3/1000 deliveries.

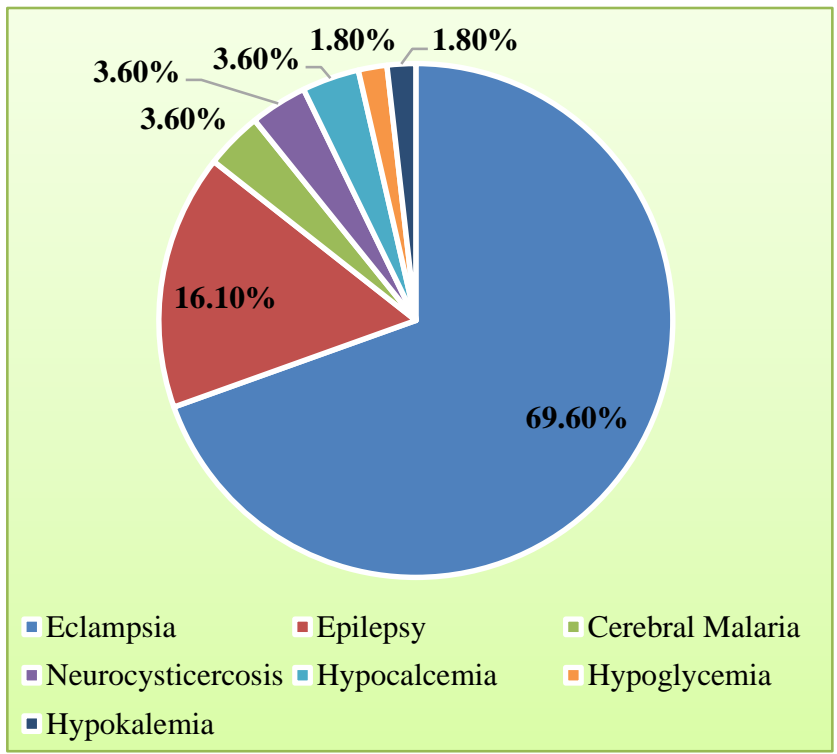

Figure 1: Etiology of convulsions in studied patient.

This was an observational study, which is based on hospital data, may underestimate or over-estimate the actual prevalence and morbidity and mortality. But it certainly gives an idea about the clinical risk factors, complications and their association with maternal and fetal outcome.

The most common aetiology was eclampsia (69.6\%). This was followed by epilepsy (16.1\%), and other causes which included cerebral malaria, neurocysticercosis, metabolic derangements like hypoglycaemia and hypokalemia. This is shown in Figure1.

Majority of patients $(41.1 \%)$ were in the age group of 21 25 years. The age distribution is shown in Table 1 . The average age was $25.12 \pm 4.99$ years. There was no significant association of age with maternal outcome.

$89.3 \%$ patients were antenatally registered. $10.6 \%$ patients were unregistered. Out of the registered patients almost $70 \%$ patients had less than 4 antenatal visits.

Table 1: Age distribution of the patients in present study.

\begin{tabular}{|lll|}
\hline Age group & Number & Percentage \\
\hline$<20$ years & 11 & 19.64 \\
\hline 20-25 years & 23 & 41.07 \\
\hline 26-30 years & 17 & 30.35 \\
\hline 31-35 years & 3 & 5.36 \\
\hline$>35$ years & 2 & 3.57 \\
\hline
\end{tabular}

The number of primigravida $(48.2 \%)$ and multigravida $(51.8 \%)$ were almost similar. $48.21 \%$ patients delivered vaginally, $51.79 \%$ patients required lower segment cesarean section overall. The difference in outcome between eclamptic and non-eclamptic patients was found to be significant (Table 2).

Table 2: The distribution of the mode of delivery.

\begin{tabular}{|lll|}
\hline Mode of delivery & Number & Percentage \\
\hline $\begin{array}{l}\text { Vaginal (Including } \\
\text { instrumental delivery) }\end{array}$ & 27 & 48.21 \\
\hline Caesarean section & 29 & 51.79 \\
\hline Total & 56 & 100 \\
\hline
\end{tabular}

A total of 15 patients had convulsions due to causes other than eclampsia. There were total 9 epileptic patients, 8 of whom were on antiepileptic drugs. Two of the patients of neurocysticercosis where on anti-epileptic medications to avoid convulsions due to CNS irritability.

Two of the three patients of metabolic abnormalities (hypokalemia and one patient of hypocalcemia) were given magnesium sulphate for seizures in addition to the metabolic correction.

A total of 6 patients needed ICU admission (4 eclampsia, 2 non-eclampsia). Overall 17 patients required blood and blood transfusions for various indications like anaemia, 
postpartum haemorrhage, DIC, HELLP syndrome etc. 14 of these had eclampsia (Table 3 and 4).

Table 3: Comparison between eclamptic and noneclamptic patients requiring ICU stay and blood and blood products transfusion.

\begin{tabular}{|c|c|c|}
\hline No. of patients & Eclampsia & $\begin{array}{l}\text { Non- } \\
\text { eclampsia }\end{array}$ \\
\hline \multicolumn{3}{|c|}{ Comparison regarding ICU stay } \\
\hline ICU stay (yes) & 4 & 2 \\
\hline ICU stay (no) & 35 & 15 \\
\hline \multicolumn{3}{|c|}{ Comparison regarding blood product need } \\
\hline $\begin{array}{l}\text { Blood products received } \\
\text { (yes) }\end{array}$ & 14 & 3 \\
\hline $\begin{array}{l}\text { Blood products received } \\
\text { (no) }\end{array}$ & 25 & 14 \\
\hline
\end{tabular}

Both the mortalities in this study were in the registered patients but with inadequate and irregular visits. Maternal case fatality rate was $3.6 \%$. It included 2 patients.

Table 4: Comparison of various clinic-demographic parameters in eclamptic and non-eclamptic patients.

\begin{tabular}{|c|c|c|}
\hline & Eclampsia & $\begin{array}{l}\text { Non- } \\
\text { Eclampsia }\end{array}$ \\
\hline \multicolumn{3}{|c|}{ Age Distribution $(p$ value $=0.2279)$} \\
\hline Age $<30$ years & 35 & 13 \\
\hline Age $>30$ years & 4 & 4 \\
\hline \multicolumn{3}{|l|}{ Parity $(\mathrm{p}$ value $=0.2553)$} \\
\hline Primigravida & 23 & 7 \\
\hline Multigravida & 16 & 10 \\
\hline \multicolumn{3}{|c|}{ Gestational age at delivery $(p$ value $=0.7719)$} \\
\hline Preterm $(<37$ weeks $)$ & 20 & 10 \\
\hline Term (>37 weeks) & 19 & 7 \\
\hline \multicolumn{3}{|c|}{ Mode of Delivery $(p$ value $=0.0415$ ) } \\
\hline $\begin{array}{l}\text { Vaginal (Including } \\
\text { instrumental delivery) }\end{array}$ & 15 & 12 \\
\hline Caesarean section & 24 & 5 \\
\hline \multicolumn{3}{|c|}{ Foetal Outcome $(p$ value $=1.00)$} \\
\hline Good (Discharged) & 33 & 15 \\
\hline $\begin{array}{l}\text { Poor (IUFD/ Neonatal } \\
\text { death) }\end{array}$ & 6 & 2 \\
\hline \multicolumn{3}{|c|}{ Maternal Morbidity: ICU Stay (p value=1.0) } \\
\hline Need for ICU stay Yes & 4 & 2 \\
\hline Need for ICU stay No & 35 & 15 \\
\hline \multicolumn{3}{|c|}{ Blood products transfusion $(p$ value $=0.2518)$} \\
\hline $\begin{array}{l}\text { Need for blood and } \\
\text { blood products (yes) }\end{array}$ & 14 & 3 \\
\hline $\begin{array}{l}\text { Need for blood and } \\
\text { blood products (no) }\end{array}$ & 25 & 14 \\
\hline
\end{tabular}

Case 1: Day 2 of full-term vaginal delivery. A case of eclampsia with HELLP syndrome with DIC.

Case 2: Postpartum Day 10 of emergency LSCS done at an outside hospital with on and off fever since postoperative day 5. There was 1 episode of convulsion on day 7 post LSCS associated with peri-oral twitching. No CNS abnormalities detected. Patient was normotensive with no proteinuria. On investigating leucocyte count was high, fever profile negative, however calcium level was found to be low $(6.2 \mathrm{gm} / \mathrm{dl})$ for which correction was given. Patient died of sepsis with MODS.

$42.9 \%$ babies required NICU admission. Most of them were admitted for prematurity. Total perinatal mortality was $14.2 \%$ of which $6(10.7 \%)$ were still birth. There were $2(3.6 \%)$ neonatal deaths. There were no malformations observed.

\section{DISCUSSION}

Epilepsy forms the major cause of non-eclamptic convulsions. The overall prevalence of epilepsy in adults is $1.65 \% .^{5}$ In India, a study done by Kerala registry following up on all epilepsy patients delivered between 1998-2013 shows an approximate prevalence of 1 in 2000 pregnancies. ${ }^{6}$

\section{Age}

The mean age of patients in our study was $25.12 \pm 4.99$ years which was similar to the study by Ozdemir et al, where mean age of the patients was $27.12 \pm 5.4$ years. ${ }^{5} \mathrm{~A}$ similar preliminary result of the Kerala registry for epilepsy and pregnancy had a mean age of 26 years. $^{7}$

\section{Mode of delivery}

In the present study 24 out of the 39 patients of eclampsia required emergency lower segment caesarean section for indications like foetal distress, failure of induction, malpresentations and antepartum haemorrhage. Whereas only 5 of the 17 non-eclamptic patients needed LSCS for similar indications which was statistically significant. In the study by Ozdemir $14.76 \%$ patients had vaginal delivery while $79.20 \%$ had a caesarean section. ${ }^{6}$ While in the study by Thomas S V, almost one-third of the patients required LSCS. ${ }^{5}$

\section{Antiepileptics usage}

The American Academy of Neurology recommends optimized monotherapy over polytherapy for seizure control in patients with epilepsy since it reduces longterm neurological sequelae in the child. Newer antiepileptics like levetiracetam and lamotrigine are gaining popularity over valproate, carbamazepine, phenobarbital etc due to their lower teratogenic potential. Valproate being most teratogenic is generally avoided. However, if the seizures are only responsive to valproate, the lowest possible dose must be administered with due risks explained to the patient. Folic acid supplementation is a must in all cases. ${ }^{8}$

A study by Danielsson KC observed that valproate predisposed patients to develop mild pre-eclampsia, 
which was not observed in patients receiving lamotrigine or levetiracetam. ${ }^{9}$

\section{Malformations}

The risk of congenital malformations varies with each anti-epileptic drug. Drugs like valproate and phenobarbitone have higher teratogenic potential compared to others. The North American Anti-epileptic Drug Pregnancy Registry (NAAPR) is one of the largest registries studying the major congenital anomalies caused by anti-epileptic drugs. They found that valproate had the maximum incidence of major malformations like neural tube defects, cardiac defects etc.

Most of the patients with epilepsy in our study where on levetiracetam. There were no malformations noted in our study. This is similar to the findings of Bansal et al and International bodies like UK and Ireland registries and Australian registry. ${ }^{10,11}$ However, an increased rate of seizure recurrence was noted by Bansal et al during pregnancy in women with epilepsy on levetiracetam. This could be attributed to certain pharmcokinetics like increased renal clearance, increase in volume of distribution etc. Hence ideally a therapeutic drug monitoring should be done in all patients to avoid recurrence of seizures. The non-identification of any malformations in our study may be because of a lack of larger sample size.

\section{Neonatal outcome}

The perinatal mortality was $14.2 \%$ of which $8.7 \%$ (6 babies) were still birth. Two were $(3.6 \%)$ neonatal deaths due to extreme prematurity. Three babies had birth asphyxia. About $57 \%$ babies were low birth weight $(<2500 \mathrm{gm})$ which is almost double that of the study by Thomas S V, where $21.4 \%$ had low birth weight. While the study conducted by Ozdemir showed IUGR to be most common neonatal complication. ${ }^{5}$

\section{Prematurity}

Seizures can precipitate early labour. 17 of the babies born in our study $(30.3 \%)$ were preterm, some patients had spontaneous onset of labour while majority of them were iatrogenic due to induction of labour for maternal well-being.

\section{NICU Stay}

Overall $42.9 \%$ babies required NICU admission. Out of these majority of the babies were born to patients with eclampsia. Most had preterm delivery. Other causes for NICU admission include respiratory distress, birth asphyxia and neonatal jaundice. $39.3 \%$ were discharged while $2(3.6 \%)$ babies expired. 32 babies did not require NICU.
A "Seizure" is a transient neurological dysfunction that is secondary to abnormal, excessive and hypersynchronous neuronal discharges this may manifest physically as convulsions. Convulsions in pregnancy are always considered to be due to eclampsia unless proved otherwise. ${ }^{12}$ This discussion aims at highlighting the other causes of convulsions in pregnancy that were encountered in studied patients.

Epilepsy was the most common cause of non-eclamptic convulsions in our patients. 9 out of the 56 patients were a known case of epilepsy. 6 out of these 9 patients were on monotherapy (levetiracetam). One was on valproate, one on polytherapy (carbamazepine and levetiracetam), one patient was not on any medication. Certain antiepileptics are known to cause congenital anomalies. Many studies have concluded that the most common amongst them is Valproate. Polytherapy increases the risk further as compared to monotherapy. There were no congenital anomalies noted in any of the babies born to mothers taking anti-epileptic medication in present study.

The next common cause that was noted was electrolyte imbalance which could lead to various manifestations, convulsions being a common presenting symptom. Conditions like hypocalcemia, hypoglycaemia, hyper or hyponatremia can all lead to convulsions. We had two (3.6\%) patients with hypocalcemia related convulsions. Hypocalcemia may be caused due to hypoparathyroidism, vitamin D deficiency, chronic kidney failure, pancreatitis or at times medication related. Ali et al describe a case report of convulsions precipitated by hypo-calcemia in an epileptic patient on long term anti-epileptic treatment. ${ }^{13}$ This occurs due to vitamin D deficiency which further leads to hypocalcemia. There was no organic pathology identified in both our patients, hence it was presumed to be due to idiopathic hypoparathyroidism which was corrected by intravenous calcium therapy. However, one of the patient developed sepsis with MODS and ultimately succumbed to death. Hypokalemia is a rare cause of convulsion not much literature is available for the same. The exact cause of hypokalemia could not be determined in participated patient.

Diabetes in pregnancy is a rising epidemic. An extremely high rate of severe hypoglycaemia, ranging between $19 \%$ and $44 \%$ has been reported in pregnancies complicated by diabetes. ${ }^{14}$ This severe hypoglycaemia can cause convulsions and at times even coma. There was one patient in our study who had convulsion due to hypoglycaemia. She was a known case of diabetes since 4 years compliant on insulin treatment. However, she had a brief history of fasting on the day she had the convulsion. The glucose level of this patient at the time of convulsion was $47 \mathrm{mg} / \mathrm{dl}$.

The second common condition noted in our patients was cerebral malaria and hypocalcemia. Cerebral malaria is a severe form of malaria caused by Plasmodium falciparum parasite. Cerebral malaria is a fatal condition. Two 
(3.6\%) of our patients were diagnosed with the same. Severe anaemia is an important and frequent consequence of severe falciparum infection which was noted in both our cases. Thrombocytopenia being another grave lifethreatening complication was also noted in one patient. Overall, besides convulsions, features like anaemia, jaundice, pulmonary oedema, ARDS, stillbirth, preterm labour and increased morbidity and mortality have been noted. The management must be multidisciplinary and in an intensive care unit. The role of corticosteroids or mannitol in treatment of cerebral oedema in a case of cerebral malaria is controversial.

A recent Cochrane database systemic review does not recommend the routine use of mannitol or other osmotic diuretics in the adjunctive treatment of cerebral malaria. ${ }^{15}$ WHO recommends the use of parenteral artemisinin derivatives as the first-line treatment of severe malaria however, resistance is an emerging problem hence Quinine is commonly used a first line management in uncomplicated cases. ${ }^{16,17}$

Neurocysticercosis is one of the uncommon causes of convulsions. Two (3.6\%) of studied patients were diagnosed with neurocysticercosis. The possibility of neurocysticercosis should especially be considered in immigrant travellers. Neurocysticercosis is a parasitic infection caused by larvae of Tapeworm Taenia Solium. ${ }^{18}$ These infections are highest in developing countries where multiple factors like poor sanitation, slaughtering of pigs, bad cooking habits; and poor drainage of waste continues the cycle of contamination. MRI may show ring enhanced lesions which forms an accurate means of diagnosing the condition. Calcified lesions may be seen on plain as well as contrast studies. However, these need to be differentiated from conditions like tuberculoma.

There has been no research to study the impact of this parasitic infection on maternal and fetal well-being due to the paucity of cases reported in pregnancy. Some studies have reported that pregnancy related changes can cause reactivation of the parasite. ${ }^{19} \mathrm{~A}$ case reported by Sahai et al states that neurocysticercosis should be considered as a differential diagnosis in patients presenting with signs and symptoms of raised intracranial tension. ${ }^{20}$ The viability of the parasite decides the line of treatment. If the parasite is dead, it may act as an epileptogenic focus, hence patient needs to be given anticonvulsants. However, if there is a viable parasite an initial course of steroids and immune-suppressants needs to be given. Often, dexamethasone is given at dose range of 4.5 and $12 \mathrm{mg} /$ day. Prednisone at $1 \mathrm{mg} / \mathrm{kg} /$ day is preferred when long-term steroid therapy is required. This is usually followed by antihelminthic medications which include Albendazole $15 \mathrm{mg} / \mathrm{kg} /$ day a month or Praziquantel $50 \mathrm{mg} / \mathrm{kg} /$ day in divided doses for 15 days. ${ }^{21,22}$

In the first week of anti-helminthic treatment, the death of the parasite may lead to an exacerbation of neurological symptoms due to the local inflammation. Hence, generally anti-helminthic treatment is supplemented with steroids to control the oedema and raised intracranial tension that may occur as a result of therapy. Albendazole is less expensive, has better penetration into cerebrospinal fluid and its concentrations are not affected when given with steroids. $^{23}$

Although eclampsia formed the bulk of the cases, other non-eclamptic cases were also encountered in a significant number.

\section{ACKNOWLEDGMENTS}

Authors would like to thank Dr. Madhva Prasad for his support during study and valuable inputs for formatting the manuscript.

Funding: No funding sources

Conflict of interest: None declared

Ethical approval: The study was approved by the Institutional Ethics Committee

\section{REFERENCES}

1. Douglas KA, Redman CWG. Eclampsia in the United Kingdom. The "BEST" way forward. Br J Obstet Gynaecol. 1992;99:355-6.

2. National Institute for clinical excellence. The Epilepsies. The diagnosis and management of the epilepsies in adult and children in primary and secondary care. Clinical Guideline 20. London: NICE, 2004.

3. Yerby MS. Quality of life, epilepsy advances, and the evolving role of anticonvulsants in women with epilepsy. Neurol. 2000;55(5 suppl 1):S21-S31; discussion S54-S5814.

4. Hvas C, Henriksen T, Ostergaard J, Dam M. Epilepsy and pregnancy: effect of antiepileptic drugs and lifestyle on birth a weight. Br J Obstet Gynaecol. 2000;107:896-902.

5. Ozdemir O, Sari ME, Kurt A, Sakar VS, Atalay CR. Pregnancy outcome of 149 pregnancies in women with epilepsy: Experience from a tertiary care hospital. Interv Med Appl Sci. Academic Publishing House. 2015 Sep;7(3):108-13.

6. Thomas S V., Jose M, Divakaran S, Sankara Sarma P. Malformation risk of antiepileptic drug exposure during pregnancy in women with epilepsy: Results from a pregnancy registry in South India. Epilepsia. 2017 Feb;58(2):274-81.

7. Thomas S V, Indrani L, Devi GC, Jacob S, Beegum J, Jacob PP, et al. Pregnancy in women with epilepsy: preliminary results of Kerala registry of epilepsy and pregnancy. Neurol India. 2001 Mar;49(1):60-6.

8. Maheshwari A, Athale S, Lekhra OP. Comparative safety of antiepileptic drugs during pregnancy. Neurology. 2013;80(7):689. 
9. Patel SI, Pennell PB. Management of epilepsy during pregnancy: an update. Ther Adv Neurol Disord. SAGE Publications; 2016;9(2):118.

10. Danielsson KC, Borthen I, Morken N-H, Gilhus NE. Hypertensive pregnancy complications in women with epilepsy and antiepileptic drugs: a populationbased cohort study of first pregnancies in Norway. BMJ Open. 2018 Apr 24;8(4):e020998.

11. Bansal R, Suri V, Chopra S, Aggarwal N, Sikka P, Saha SC, et al. Levetiracetam use during pregnancy in women with epilepsy: Preliminary observations from a tertiary care center in Northern India. Indian J Pharmacol. 2018;50(1):39-43.

12. Mawhinney E, Craig J, Morrow J, Russell A, Smithson WH, Parsons L, et al. Levetiracetam in pregnancy Results from the UK and Ireland epilepsy and pregnancy registers. Neurol. 2013 Jan 22;80(4):400-5.

13. Lowenstein D. H. seizures and epilepsy in harrisons principles of internal medicine Volume 2 (Eds) Fauci. A. S. Braunwald E. Isselbacher K. J. et al, $14^{\text {th }}$ ed. McGrew Hill New York, 1998, 2311-2325.

14. Ali FE, Al-Bustan MA, Al-Busairi WA, Al-Mulla FA. Loss of Seizure Control Due to AnticonvulsantInduced Hypocalcemia. Ann Pharmacother. 2004 Jun;38(6):1002-5.

15. Persson B, Hansson U. Hypoglycaemia in pregnancy. Baillière's Clin Endocrinol Metab. 1993 Jul ;7(3):731-9.

16. Tjitra E, Anstey NM, Sugiarto P, Warikar N, Kenangalem E, Karyana M, et al. Multidrugresistant Plasmodium vivax associated with severe and fatal malaria: a prospective study in Papua, Indonesia. PLoS Med. 2008;5:e128.
17. Brabin BJ, Eggelte TA, Parise M, Verhoeff F. Dapsone therapy for malaria during pregnancy. Drug Safety. 2004 Aug 1;27(9):633-48.

18. Valecha N, Pinto RG, Turner GD, Kumar A, Rodrigues S, Dubhashi NG, et al. Histopathology of fatal respiratory distress caused by Plasmodium vivax malaria. Am J Trop Med Hyg. 2009;81:75862.

19. Maiti B. Neurocysticercosis an update. JAPI. 1995;43:477-87.

20. D'Cruz RF, Ng SM, Dassan P. Neurocysticercosis in pregnancy: maternal and fetal outcomes. Oxford Med Case Reports. 2016 Jul;2016(7):138-40.

21. Sahai S, Sahai A. Pica causing neurocysticercosis in pregnancy presenting as eclampsia: a report of two cases. J Obstet Gynaecol India. 2013 Mar;63(1):689.

22. Nutman B, Thomas B., Weller P.F., Cestodes in Harrisons Principles of Internal Medicine, Vol 1, (Eds) Fauci A S Braunwald E, Isselbacher K.J., Wilson J.D. et al. $14^{\text {th }}$ ed, McGraw Hill, New York, 1998:1224-1229.

23. Nash TE. Neva FA. Recent Advances in diagnosis and treatment of Neurocysticercosis N Eng J Med. 1994;311:1492-6.

Cite this article as: Fonseca M, Joshi A. Maternal and fetal outcome of pregnancy complicated by convulsions. Int J Reprod Contracept Obstet Gynecol 2018;7:3801-7. 